\title{
Coming of age: emergency point of care ultrasonography in Canada
}

\author{
Paul Atkinson, MB, BCh, BAO, MA*; Peter Ross, MD*; Ryan Henneberry, MD†
}

In the spring of 2012, the Canadian Association of Emergency Physicians (CAEP) issued an important revised position statement outlining its support for point-of-care ultrasonography (PoCUS) by emergency physicians in the emergency department. ${ }^{1}$

Emergency PoCUS was born in Canada in 2001: Emergency department echocardiography was a child of the international emergency ultrasound movement that traces its origins back to the late 1980s in the United States. ${ }^{2}$ The Canadian Emergency Ultrasound Society (CEUS) has been the only organization providing formal certification for the practice of emergency ultrasonography in Canada over the past decade. We support the continued growth of emergency ultrasonography in Canada and call for a renewed effort to ensure that it becomes a universal skill for all emergency physicians.

Definitions of emergency ultrasonography may vary depending on the clinical indications; however, most emergency physicians would describe it as "diagnostic or procedural ultrasound that is performed and interpreted by the emergency physician during the initial patient encounter for the evaluation of emergent conditions."

The use of PoCUS as an adjunct to the practice of emergency medicine (EM) is now well established both internationally and in Canada. Initially, evidence to support using PoCUS came from experiences managing patients who had sustained blunt trauma. ${ }^{3}$ However, the scope of practice has expanded as emergency physicians have identified further clinical problems where PoCUS is able to aid evaluation and procedures. Core applications include identification of abdominal aortic aneurysm, focused echocardiography in cardiac arrest, and identification of an intrauterine pregnancy in the first trimester, in addition to trauma. More recently, the scope of the answers provided by PoCUS has expanded to include such problems as identification of pleural fluid and pneumothorax, identification of a deep vein thrombosis, assessment of the inferior vena cava for filling status, shock protocols, and more traditional ultrasound indications, such as cholelithiasis and hydronephrosis. ${ }^{1}$ Ultrasound guidance makes insertion of central venous catheters safer and improves success rates for regional nerve blocks. ${ }^{4-7}$

In everyday practice, the emergency physician regularly faces challenges trying to identify serious pathology or reduce procedural complications in a timely manner. Necessity is the mother of invention, and as a result, emergency physicians have continued to develop innovative uses for and expanded the indications for PoCUS. It should be noted that the American College of Emergency Physicians (ACEP) has recently classified emergency ultrasonography into five functional clinical categories to more clearly distinguish its various uses:

- Resuscitative: ultrasonography used as directly related to an acute resuscitation

- Diagnostic: ultrasonography used in an emergent diagnostic imaging capacity

- Symptom or sign based: ultrasonography used in a clinical pathway based on the patient's symptom or sign (e.g., shortness of breath)

- Procedure guidance: ultrasonography used as an aid to guide a procedure

From the *Department of Emergency Medicine, Dalhousie University, Saint John Regional Hospital, Saint John, NB; and tHalifax Infirmary, Halifax, NS.

Correspondence to: Dr. Paul Atkinson, Emergency Department, Saint John Regional Hospital, University Avenue, Saint John, NB E2L 4L4; Paul. Atkinson@dal.ca.

This article has been peer reviewed.

(C) Canadian Association of Emergency Physicians 
- Therapeutic and monitoring: ultrasonography used in therapeutics or in physiologic monitoring ${ }^{8}$

A key feature of PoCUS is that it is not a replacement to traditional ultrasound practice but a limited ultrasound examination usually practiced at the bedside of the patient, in suboptimal conditions with time limitations. Hence, the application is focused to answer a clinical question (or set of questions), which can augment clinical care. As such, PoCUS practice has also moved into areas not traditionally examined by ultrasonography, including the sonographic diagnosis of certain pulmonary pathologies, such as interstitial and alveolar fluid. ${ }^{9}$ As ultrasonography matured within EM and its practitioners developed relationships with physicians outside the EM family, it developed several new aliases, such as emergency department targeted ultrasonography, emergency and critical care ultrasonography, focused emergency ultrasonography, and now, more universally, PoCUS. ${ }^{?}$

PoCUS now transcends specialty boundaries, has relevance to most clinician groups, and is becoming integrated into undergraduate medical education in some centres. ${ }^{9,10}$ Popularity of use has also been influenced by improved and tailored ultrasound systems: reduction in price and relative size, improved portability and quality, ease of use, and speedy start-up times. We encourage all physicians to review Moore and Copel's list of current PoCUS applications in use within various clinical specialties. ${ }^{9}$ With a plethora of PoCUS applications available, there are continued challenges to ensure that such PoCUS can be delivered with adherence to good governance principles.

Now that ultrasound practice has matured, it is important that the Canadian emergency PoCUS family carefully considers the next steps in its life story. With the Royal College of Physicians and Surgeons of Canada (RCPSC), the College of Family Practice of Canada (CFPC), CEUS, and CAEP recommending ultrasound training as a core curricular competency, it is essential that opportunities for PoCUS training are available as part of all residency programs across the country.

Currently, there is variability in both the level and the quality of PoCUS training in Canadian EM residency programs, with a majority of residents using educational resources outside their residency training to supplement their ultrasound knowledge. ${ }^{11}$ Effort must be made to ensure rigorous standardization of training across residencies so that the requirement of an additional certificate in PoCUS, having obtained $\mathrm{CCFP}(\mathrm{EM})$ or $\mathrm{FRCP}(\mathrm{EM})$, can become a thing of the past. In this way, PoCUS skill acquisition will join the other EM core skills, such as airway management, electrocardiography, and radiographic interpretation, which currently require no such additional certification.

To begin to progress toward this goal, we must consider the following:

- Training. Although the traditional model of an introductory course or modules, followed by a number of supervised determinate scans, may continue in the foreseeable future, a multifaceted approach is needed for the following reasons: some pathology conditions will be seen infrequently; there may be a paucity of trained supervisors in certain locales; and one-to-one supervision is not always possible. Additional training models could include simulation and Web-based training models, both of which are becoming more established and more popular. ${ }^{12,13}$ PoCUS skills are critical to the clinical development of an emergency physician, and a minimum skill set should be mandatory for all graduating EM residents. ${ }^{14,15}$ The ultrasound education provided to EM residents should be structured to allow residents to incorporate ultrasonography into daily clinical practice. ${ }^{16}$

- Credentialing. It is essential to ensure that a trainee in PoCUS has demonstrated competency in a particular application before he or she can practice independently. There is evidence to demonstrate that competency can be assumed after a number of ultrasound examinations have been undertaken, although in practice, not all logged examinations are of the same quality. ${ }^{8}$ Others favour the use of triggering a competency assessment after a recommended period of training. Such an assessment is undertaken against a number of objective criteria and is based less on the number of logged scans. Being competent to practice is not just about being able to undertake the application examination but, importantly, about understanding the relevance of what the findings mean to clinical practice. ${ }^{17,18}$ Canadian programs that graduate residents in EM via both routes, $\mathrm{CCFP}(\mathrm{EM})$ and $\mathrm{FRCP}(\mathrm{EM})$, should ensure that they provide certification of competence, at least in all core or basic PoCUS applications. ${ }^{1}$

- Governance. Once independently practicing, emergency physicians need to ensure that they keep their 
skills up to date. Hiatuses in performing a particular application may result in a loss of that skill. Peer review and audit have an important role in demonstrating continued competency. Keeping up to date with regard to the latest medical research in this area is essential. Regular maintenance of equipment and quality assurance review of ultrasound performance are required.

- Research. Although there is a growing evidence base supporting the benefits of PoCUS in EM, much of the literature to date falls short of proving true outcome benefit. ${ }^{8}$ It is important that we meet the challenge to conduct well-designed studies to further support our practice. National EM and critical care medicine organizations should lead by providing the networking opportunities required to develop large-scale, well-designed prospective studies and databases.

- Fellowships. Countries such as the United States have multiple PoCUS fellowships available to emergency physicians. ${ }^{19}$ The development of a similar Canadian network of PoCUS fellowships, with a national curriculum, based on a needs assessment, would provide and ensure leaders, researchers, and educators future excellence in emergency PoCUS.

- International relationships. PoCUS continues to evolve internationally, and as such, we in Canada need to be part of the international community that drives this growth. Organizations such as the International Federation of Emergency Medicine (IFEM) and WINFOCUS provide forums where faculty and trainees can share knowledge and skills. In time, an international curriculum and standard of practice are likely. It is important that Canadian emergency PoCUS leaders engage fully with our colleagues around the world in developing such standards.

For now, we in Canada should congratulate emergency ultrasonography on its coming of age. We must recognize that the PoCUS family is growing both within our specialty and with others, and we must ensure appropriate training in PoCUS for all physicians who make time-critical patient care decisions where we know ultrasonography makes a difference.

Competing interests: Paul Atkinson chairs the Canadian Association of Emergency Physicians (CAEP) point of care ultrasound research subcommittee, and also represents CAEP on the International Federation for Emergency Medicine (IFEM) point of care ultrasound curriculum development working group. He is a codirector and faculty member for the
Emergency Critical Care Ultrasound (ECCU) course; and faculty member for the Emergency Department Targeted Ultrasound (EDTU) course. Peter Ross is president of the Canadian Emergency Ultrasound Society (CEUS) and is also a faculty member for the Emergency Critical Care Ultrasound (ECCU) course; and for the Emergency Department Echo (EDE) course. Ryan Henneberry chairs the CAEP Ultrasound Committee and is also a faculty member for the Emergency Critical Care Ultrasound (ECCU) course; and for the Emergency Department Targeted Ultrasound (EDTU) course.

\section{REFERENCES}

1. Henneberry RJ, Hanson A, Healy A, et al. Use of point of care sonography by emergency physicians. Canadian Association of Emergency Physicians position statement. CFEM 2012;14:106-12.

2. Jehle D, Davis E, Evans T, et al. Emergency department sonography by emergency physicians. Am $\mathcal{f}$ Emerg Med 1989;7:605-11, doi:10.1016/0735-6757(89)90283-0.

3. Boulanger BR, McLellan BA, Brenneman FD, et al. Prospective evidence of the superiority of a sonography based algorithm in the assessment of blunt abdominal injury. 7 Trauma 1999;47:632-37, doi:10.1097/00005373-19991000000005 .

4. Making bealth care safer: a critical analysis of patient safety practices. Prepared for: Agency for Healthcare Research and Quality. Prepared by: University of California at San Francisco (UCSF) - Stanford University Evidence-based Practice Center. Available at: www.ahrq.gov (accessed March 18, 2013).

5. Atkinson P, Boyle A, Robinson S, Campbell-Hewson G. Should ultrasound guidance be used for central venous catheterization in the emergency department? Emerg Med $\mathcal{J}$ 2005;22:158-64, doi:10.1136/emj.2003.011288.

6. Leung J, Duffy M, Finckh A. Real-time ultrasonographically guided internal jugular vein catheterization in the emergency department increases success rates and reduces complications: a randomized, prospective study. Ann Emerg Med 2006;48:540-7, doi:10.1016/j.annemergmed.2006.01.011.

7. Gray A. Ultrasound-guided regional anaesthesia: current state of the art. Anesthesiology 2006;104:368-73, doi:10.1097/ 00000542-200602000-00024.

8. American College of Emergency Physicians. Emergency ultrasound guidelines 2008. Available at: http://www.acep.org (accessed March 18, 2013).

9. Moore CL, Copel JA. Point of care ultrasonography. $N$ Engl 7 Med 2011;364:749-57, doi:10.1056/NEJMra0909487.

10. Wong I, Jayatilleke T, Kendall R, Atkinson P. Feasibility of a focused ultrasound training programme for medical undergraduate students. Clin Teach 2011;8:3-7, doi:10. 1111/j.1743-498X.2010.00416.x.

11. Kim DJ, Theoret J, Woolfrey K, Kendall JL. Experience with emergency ultrasound training by Canadian emergency medicine residents: a national survey. CFEM 2012;14(S1):ID 5510 . 
12. Sidhu HS, Olubaniyi BO, Bhatnagar G, et al. Role of simulation-based education in ultrasound practice training. 7 Ultrasound Med 2012;31:785-91.

13. Filippucci E, Meenagh G, Epis O, et al. E-learning in ultrasonography: a web-based approach. Ann Rheum Dis 2007;66:962-5, doi:10.1136/ard.2006.064568.

14. Heller M, Mandavia D, Tayal V, et al. Residency training in emergency ultrasound: fulfilling the mandate. Acad Emerg Med 2002;9:835-9, doi:10.1111/j.1553-2712.2002. tb02174.x.

15. Reardon R, Heegaard B, Plummer D, et al. Ultrasound is a necessary skill for emergency physicians. Acad Emerg Med 2006;13:334-6, doi:10.1111/j.1553-2712.2006. tb01702.x.
16. Lanoix R, Leak LV, Gaeta T, et al. A preliminary evaluation of emergency ultrasound in the setting of an emergency medicine training program. Am 7 Emerg Med 2000;18:41-5, doi:10.1016/S0735-6757(00)90046-9.

17. Canadian Emergency Ultrasound Society. CEUS recommended standards. Available at: http://ceus.ca/008-position_statements/ 008-01.advanced_applications.htm (accessed March 18, 2013).

18. College of Emergency Medicine Ultrasound Subcommittee. Emergency medicine ultrasound, level 1 training. Available at: http://www.collemergencymed.ac.uk/Training-Exams/Training/ Ultrasound\%20training/default.as (accessed March 18, 2013).

19. EUS fellowships. Available at: http://eusfellowships.com/ programs.php (accessed March 18, 2013). 